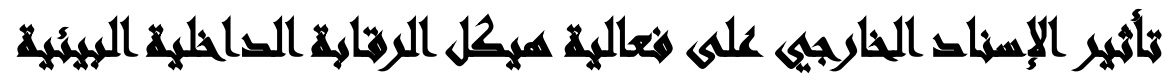

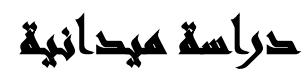

[ri $]$

محمد عبد الفتاح محدد(')- داليا عادل(')- صفاء محمود عبد الحليم

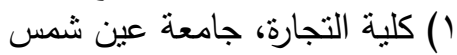

\section{المستخلمى}

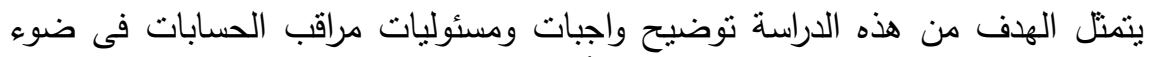

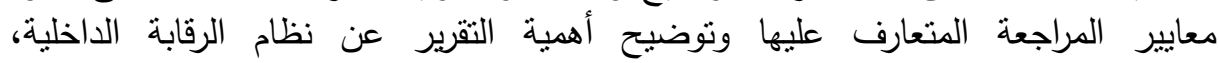

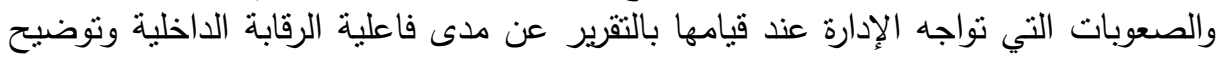

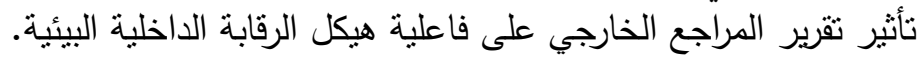

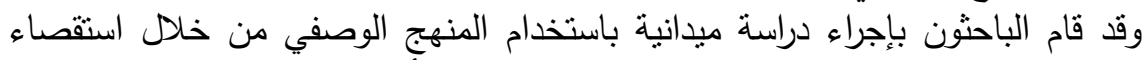

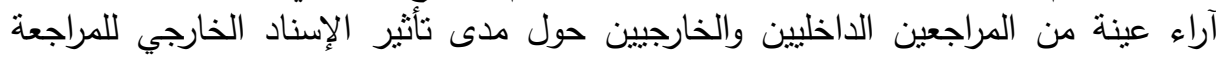
على فعالية هيكل الرقابة الداخلية البيئية.

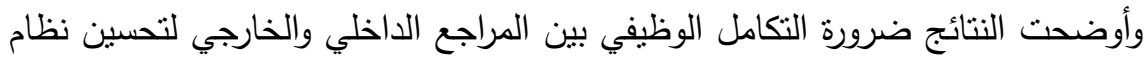

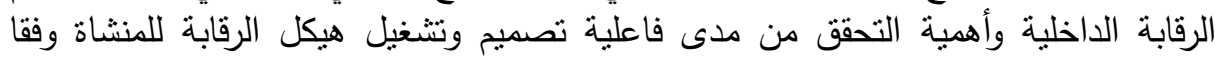

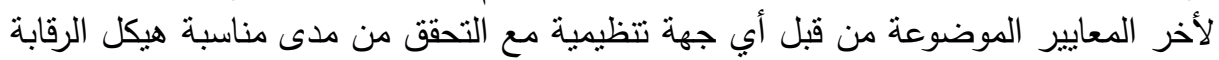

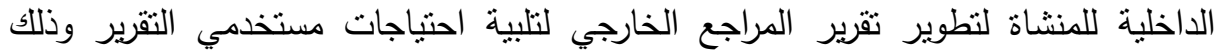

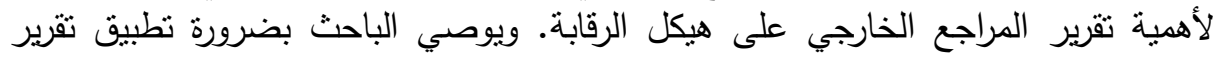
المراجع الخارجي من قبل الإدارة العليا.

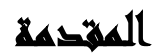

ازداد الاهتمام الدولي والاقليمى بقضايا البيئة الناجمة عن التخوف من مخاطر تدهور البيئة الطبيعية وتأثيرها على البشرية، وقد بدأت منظمات حماية البيئة في العديد من دول

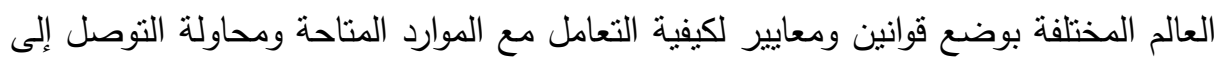
حلول للتغلب على المشكلات التي تواجه المنشآت عند تقريرها عن أدائها البيئي وتحميل المنشأة تكاليف المحافظة على البيئة.

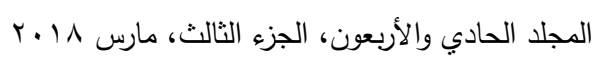


ونتيجة لللك بدأت الضغوط نتزايد على المنشآت من مختلف الفئات من مستثمرين

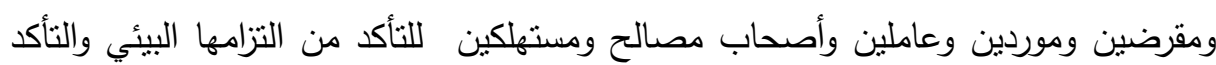
من نظم الرقابة ومدى فاعليتها لتحقيق التوازن بين أهدافها وأهمها كفاءة وفاعلية الأداء.

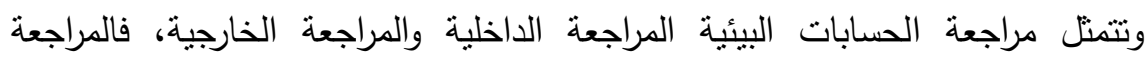
الداخلية يتولاها موظفون مدربون من المنشأة ويتطلب القيام بالمراجعة الداخلية للحسابات

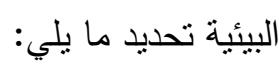
• نطاق عملية المراجعة والجدول الزمني للتنفيذ والأساليب التي تستخدم. • أعضاء فريق المراجعة ودور ومسؤولية كلا منهم ومؤهلاتهم وخبراتهم. • شكل تقرير مراجعة الحسابات والجدول الزمني لاستعراض وتقديم المعلومات المتعلقة

$$
\text { بنتائج المراجعة. }
$$$$
\text { • • المسؤولية عن الإبلاغ بنتائج عملية المراجعة. }
$$$$
\text { • المسؤولية عن اتخاذ الإجراءات الوقائية والتصحيحية. }
$$

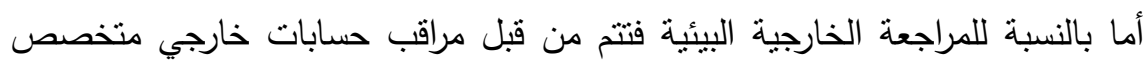
وتهدف للتأكد من توافق الأداء البيئي للمنشاة مع المعايير الوطنية والإقليمية والدولية، والمتطلبات القانونية ومتطلبات نظام الإدارة البيئية السليم، هذا بالإضافة للتأكد من صحة الإنة

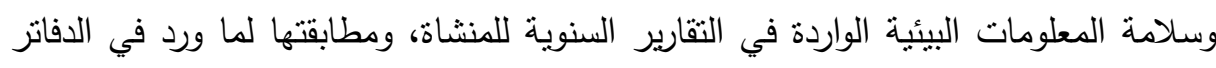
والسجلات المحاسبية، وإعدادها في ضوء المبادئ والمعايير المحاسبية المتعارف عليها

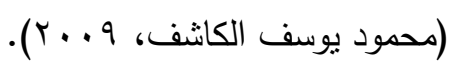

وانطلاقا من ذلك بدأ الاهتمام بالتأكد من مدى فاعلية هيكل الرقابة من خلال التأكد من

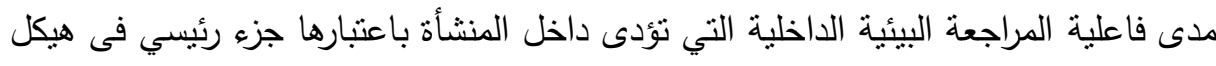
الرقابة في المنشأة ذات النشاط البيئي العالي أو من خلال الارتباط بأطراف خارجية نسند إليهم عملية المراجعة سواء جزئيا أو كليا. 


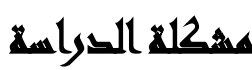

بالرغم من الإدراك المتزايد عالميا ومحليا بأهمية الحفاظ على البيئة من مخاطر التلوث والتنف البيئي تواجه المنشآت مسئوليات عديدة للوفاء بمنطلبات التنمية والحفاظ على البيئة البئة

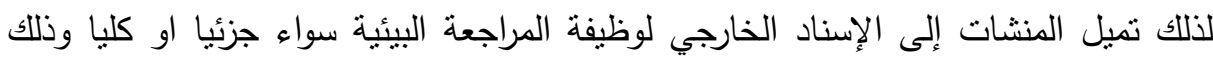

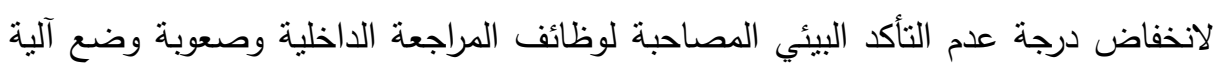

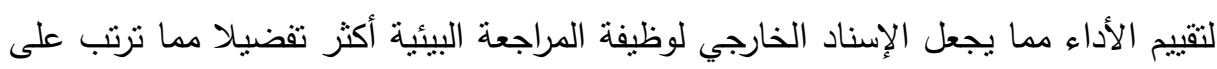

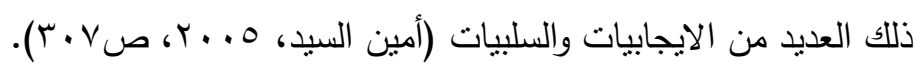

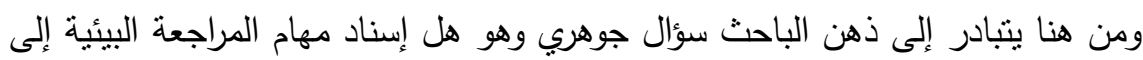

مراقب حسابات خارجي سيؤثر على فعالية الرقابة الداخلية

\section{أسريلا المهيه}

• هل تطبيق معايير المراجعة البيئية يؤدي إلى ننائج حقيقية في عملية المراجعة البيئية؟

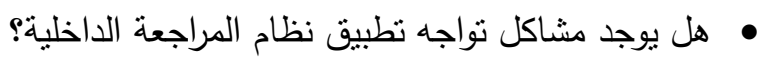

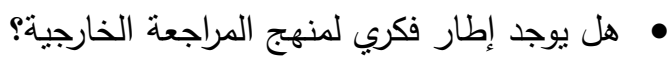

• هل تطبيق معايير المراجعة البيئية بطريقة سليمة دليل على كفاءة المراجع البيئي وخبرته؟

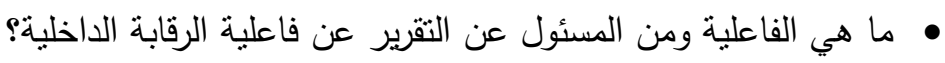

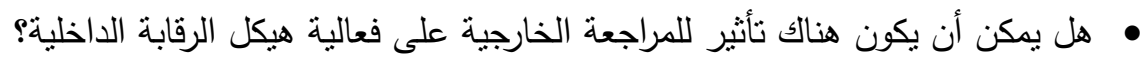

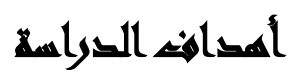

يتمتل هدف البحث الأساسى في محاولة التعرف على أثن الإسناد الخارجي للمراجعة

البيئية على هيكل الرقابة الداخلية، بالإضافة لعدة أهداف فرعية منها:

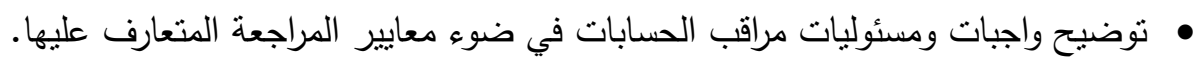

• توضيح أهية التقرير عن نظام الرقابة الداخلية، والصعوبات التي تواجه الإدارة عند قيامها

بالثقرير عن مدى فاعلية الرقابة الداخلية.

• • توضيح مدى مسئولية مراقب الحسابات عن تقرير الإدارة عن فعالية هيكل الرقابة الداخلية.

• • التوصل إلى نتائج الدراسة وتوصياتها وحل المشكلة.

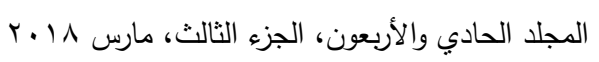




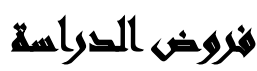

• الفرض الأول: لا توجد فروق ذات دلالة معنوية بين إدراك فئات الدراسة لأهمية الإسناد

الخارجي في الثكل الحالي التقرير المراجع الخارجي لتلبية احتباجات مستخدمي التقارير . • الفرض الثاني: لا نوجد فروق ذات دلالة معنوية بين آراء فئات الدراسة حول تأثثير تقرير

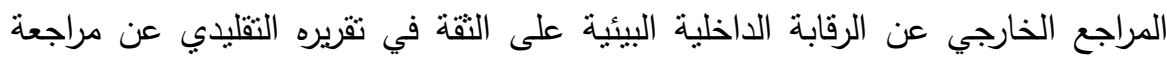
البنود. •الفرض الثالث: لا توجد فروق ذات دلالة معنوية بين آراء فئات الدراسة حول تأثثر تقرير

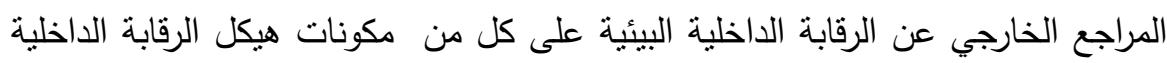
في بيئة الأعمال المصرية.

\section{أهمية التصواسة}

الأهمية العلمية: تتمثل الأهية العلمية في محاولة تأصيل المفهوم العلمي "للمراجعة المتكاملة" باعتبارها أحد المقومات التي لا يمكن إغفال أثرها على كل من تفعيل ضوابط هيكل الرقابة الداخلية وتطوير تقرير المراجع الخارجي ومن ثم تحقيق فعالية الاتصال والحد من فجوة

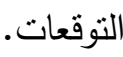
ندرة الدراسات والأبحاث التي تتاولت الإسناد الخارجي للمراجعة البيئية واثز الإسناد على هيكل الرقابة الداخلية في بيئة الأعمال المصرية. الاهمية العملية: يستمد البحث أهميته العملية من أهمية دور المراجعة البيئية في توفير قاعدة بيانات لدى القائمين بإدارة شُئون البيئة تقيد في وضع السياسات والاستراتيجيات البيئية

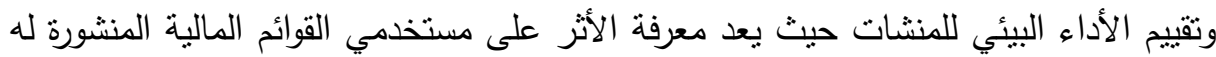
انعكاسا للمدى الذي وصلت إليه مهنة المحاسبة والمراجعة، ومدى وفاء تقارير المراجع بحاجات مستخدمي القوائم المالية المنشورة؛ ومصدرا مهما من مصادر المعلومات للمعنيين بتتظيم وتطوير مهنة المحاسبة والمراجعة. 


\section{هضوض التراسما}

الحدود المكانية: سوف يعتمد الباحث في تحديد مجال التطبيق على عدد من الثركات الصناعية المساهمة والقابضة والتابعة المدرجة في سوق المال ومكاتب الخبرة الخارجية.

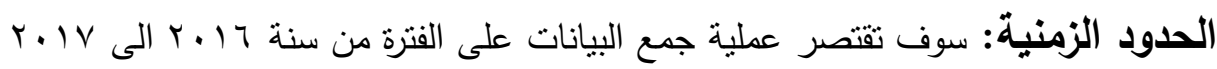
ويرى الباحث أن هذه الفترة كافية لتحقيق أهداف الدراسة. الحدود الموضوعية: واجه الباحث نقصا في الدراسات السابقة التي تتاولت العلاقة بين أهمية الإسناد الخارجي في الثكل الحالي لتقرير المراجع الخارجي وقدرته في تلبية احتباجات

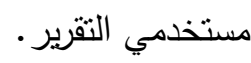

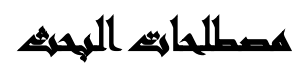

المراجعة البيئية: هي التقييم المنهجي الموثق الدوري والموضوعي لنظام الإدارة البيئية

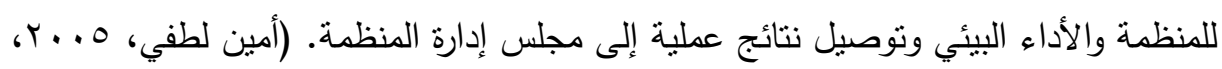

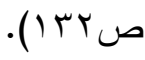
الإسناد الخارجي: هو ارتباط بين الوحدة وأطراف من خارجها لأداء خدمات تؤدى أو يمكن أدائها داخل الوحدة. (Beaumont, 2004).

\section{السراسايت المايرية}

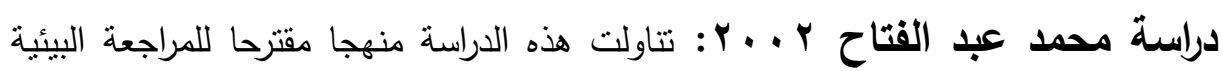

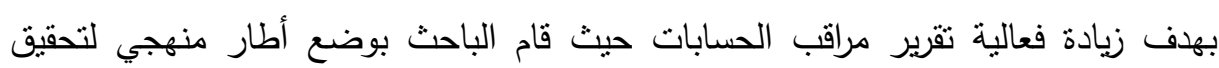
فعالية المراجعة البيئية. وأثنار إلى انه على مراقب الحسابات التحقق من وجود جهاز للمراجعة الداخلية، يحظى بتأييد الإدارة العليا.

تتاولت هذه الدراسة منهجا مقترحا للمراجعة البيئية بهدف زيادة فعالية تقرير مراقب

الحسابات حيث قام الباحث بوضع أطار منهي لتحقيق فعالية المراجعة البيئية.

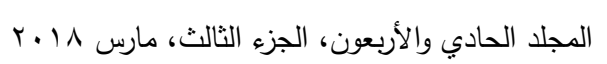


وأثنار إلى انه على مراقب الحسابات التحقق من وجود جهاز للمراجعة الداخلية، يحظى بنأييد الإدارة العليا.

وتوصلت هذه الدراسة إلى أن المراجعة البيئية تمثل أحد مجالات تكامل الجهود بين المراجع الداخلي ومراقب الحسابات، وعلى مراقب الحسابات التأكد من موضوعية المراجعة الداخلية فيما يتعلق بالاعتبارات البيئية، وذلك من خلال تقييم أوراق عمل المراجعة الداخلية، وتقييم كفاءة وموضوعية المراجع الداخلي في هذا المجال، واقتصرت الدراسة على ضرورة تحقيق مراقب الحسابات من وجود جهاز للمراجعة الداخلية تمثل مراجعة الأداء البيئي أحد مجالات أنشطته.

دراسة لأنشطة التقرير والمراجعة البيئية كهدف جوهري في الدول النامية والمتقدمة. وتم تقسيم الدراسة إلى موضوعين رئيسيين: الأول يتتاول كيفية عمل مراقب الحسابات في شركات المحاسبة والمراجعة كعضو من فريق المراجعة يؤدى أنثطة الثقرير والمراجعة موصنة البيئية. والثاني ينتاول كيفية أداء أنشطة التقرير والمراجعة البيئية من جانب مراقب لئب الحسابات بفاعلية باعتبارها هدف جوهري.

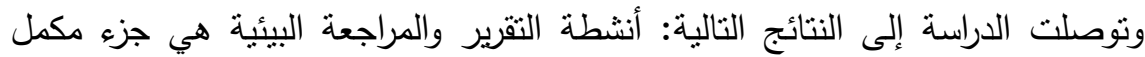
لأنظمة الإدارة البيئية ويمكن من خلالها للإدارة أن تحدد مدى كفاية أنظمة الرقابة البيئية

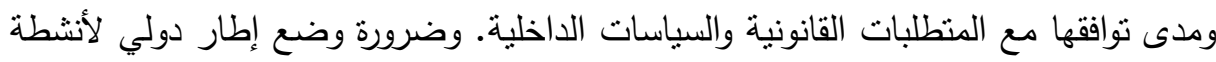
الثقرير والمراجعة البيئية لتحقيق الهدف الجوهري لهذه الأنشطة والأخذ في الاعتبار مبادئ وضئ وانئ المراجعة البيئي.

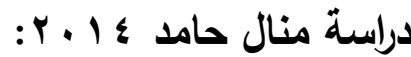
أهداف الاراسة: يتمثل الهدف الرئيسي لهذا البحث في دراسة العلاقة بين مصدر أداء وظائف

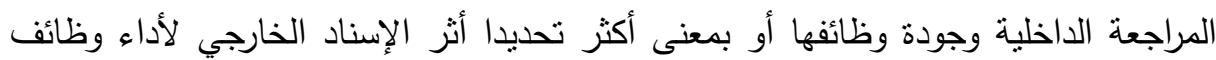
المراجعة الداخلية على جودة أداء وظائفها ويتحقق هذا الهدف من خلال الأهداف الفرعية

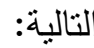


• المفاضلة بين المصادر المختلفة لأداء وظائف المراجعة الداخلية.

• • دراسة اثز الإسناد الخارجي لأداء وظائف المراجعة الداخلية على جودة أداء وظائفها.

• دراسة اثر الإسناد الخارجي لأداء وظائف المراجعة الداخلية على تخفيض تكاليف أدائها.

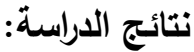

• المراجعة عملية متكررة مستمرة وليست ممارسة تتم في نقطة واحدة، وأنَّ الخبرة توضح أنَّ أفضل تتفيذ لهذه العملية هو أن تتم مشاركة بين مستشارين خارجيين وممثلين عن الوحدة المعنية لضمان الموضوعية في التحليل.

• قياس أثز عمليات المنظمة على البيئة المحيطة بها باستخدام مجموعة من المعايير

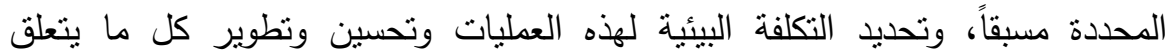
بعطيات الوحدة وأثرها على البيئة بالإضافة إلى تحديد أفضل الطرق لتطوير وتحسين هذه

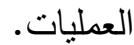

• عليات تقييم داخلية تقوم بها الشركات والجهات الحكومية للتحقق من مدى التزامها بالمتطلبات القانونية وأيضاً مدى الالتزام بسياساتها ومعاييرها الداخلية، حيث تساعد إلى هلى

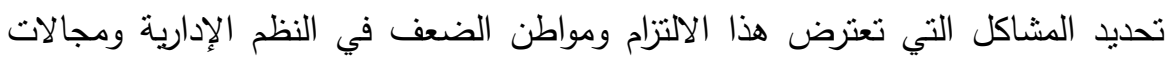

• يأخذ الدارس بمفهوم المراجعة الثاملة (مالية- قانونية- اقتصاد - كفاءة- فاعلية). • إصدار معيار المحاسبة عن المحاسبة والإفصاح عن الأحداث اللاحقة لتاريخ الميزانية.

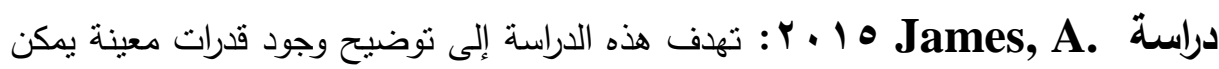
من خلالها ممارسة الدور المزدوج للمراجع الخارجي لتحقيق أقصى استفادة ممكنة وكبيرة

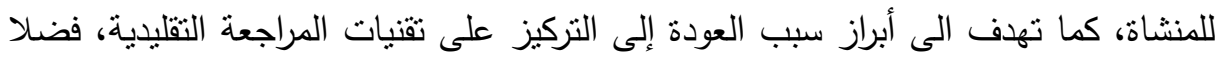
عن تقنيات المراجعة التي ترتكز على الضوابط الداخلية، وهى خطوة ضرورية للغاية، وهو يسهم في القيام بذلك في الادييات الموجودة من خلال نسليط الضوء على سبب تيسير منل

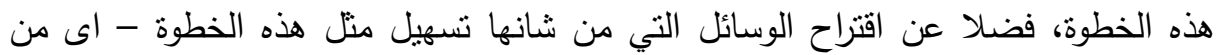

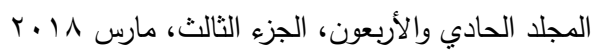


مجلة العلوم البيئية

معهد الدراسات والبحوث البيئية - جامعة عين شمس البه

خلال التركيز على الفوائد التي يمكن استخلاصها عندما يكون المراجع الخارجي قادر لادراج بعض مسئوليات المراجعة الداخلية للحسابات .

وبينما يتم التأكد على فوائد وإمكانيات الأدوار المزدوجة التي يقوم بها المراجعين

الخارجيين، فضلا عن الحاجة للتوصل إلى حلول وسط لـالئ

\section{الإطار اللنظليه}

يعتبر الإسناد الخارجي للمراجعة البيئية هو ارتباط بين الوحدة وأطراف من خارجها لأداء

خدمات تؤدى أو يمكن أدائها داخل الوحدة. (Beaumont, 2004)

يعتبر وجود علاقة تعاونية بين المراجعة الداخلية والمراجعة الخارجية أمر مهم جدا وضروريا للطرفين وللمنشاة، لما لها من تأثثير ايجابي وفائدة كبيرة لتحقيق أهداف كلا منهما بشكل خاص، وحسن سير العمل وتحقيق الفعالية المطلوبة لتحقيق أهداف المنشاة بشكل عام، وما يؤكد على أهمية هذا التكامل ويعززه هو قيام بعض المنظمات المهنية الدئه الدولية بإصدار معايير تحكم وتتظم العديد من أوجه التكامل بين المراجعة الداخلية والخارجية. لللك يسعى كلا من المراجع الداخلي ومراقب الحسابات الخارجي إلى التأكد من وجود نظام فعال للرقابة الداخلية في المنثأة، من خلال دراسته وتقييمه وتحديد نقاط ضعفه وقوته، والتأكد من أن النظام المحاسبي المتبع فعال، ويمد المنشأة بالمعلومات اللازمة لإعداد القوائم المالية الصحيحة التي يمكن الاعنماد عليها لاتخاذ قرارات فعالة لتحقيق الأهداف المرجوة.

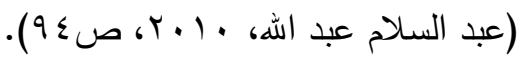

ولذلك فهناك العديد من الدواعي للتقرير عن فعالية هيكل الرقابة الداخلية ومن أهمها:

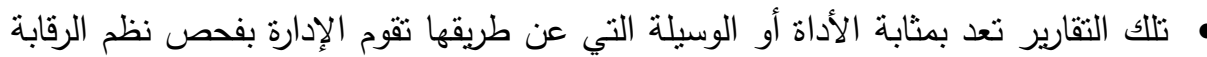
الداخلية للمنشأة. • تلك التقارير توفر لمستخدمي القوائم المالية قاعدة يمكن الاعتماد عليها في تقييم نظم الرقابة الداخلية بالمنشاة حيث يعتبر تقرير مراقب الحسابات الخارجي عن فعالية الرقابة الداخلية يمكن بواسطته تحديد أي مخاطر يمكن أن تتعرض لها المنشأة. • بواسطة تلك الثقارير يمكن تحديد مدى أمكانية الاعتماد على هيكل الرقابة الداخلية. 
ووفقا للمعيار رقم (r) الصادر عن PACOB فان مراقب الحسابات يمكنه أبداء رأيه في تقرير الإدارة عن مدى فاعلية نظام الرقابة الداخلية، وذلك بعد تقييمه للأدلة التي أمكنه

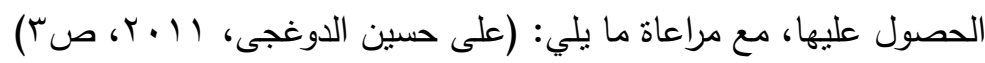
• أن يكون الرأي في الرقابة الداخلية يغطى سنة ماعل الية.

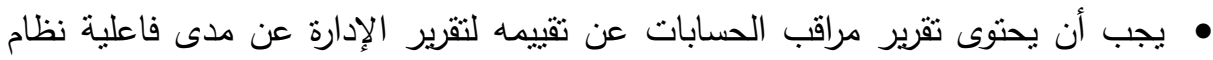
الرقابة الداخلية عن القوائم المالية رأيه في نظام الرقابة الداخلية.

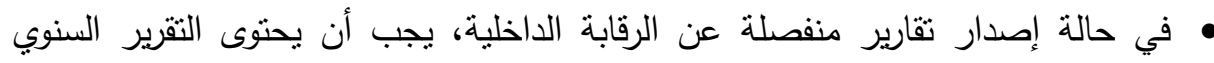
للإدارة على كليهما (تقرير عن مدى فاعلية نظام الرقابة الداخلية، وتقرير عن القوائم

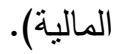

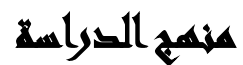

يعثمد البحث في الجانب النظري علي المنهج الاستقرائي من خلال الرجوع إلى الدوريات

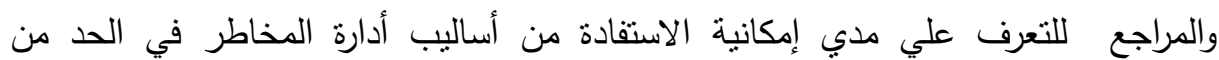
المخاطر البيئية لتحقيق الأهداف الإستراتيجية للمنشاة والاعتماد على المنهج الاسنتباطي الإسي لدراسة العلاقة بين الإسناد الداخلي والخارجي.

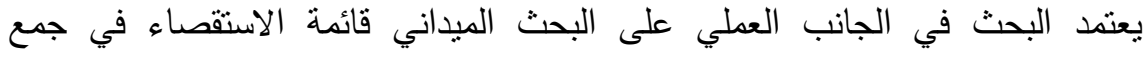
البيانات، حيث يتم إعدادها بالثكل الذي يحقق أهداف البحث، بهدف الحصول علي البيانات اللازمة لاختبار فروض البحث والتحقق من صحته.

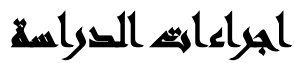

أدوات الدراسة:بعض الاطلاع على عدد من الدراسات والأبحاث ذات العلاقة بالموضوع تم تصميم استبانة تتكون من r محاور كل محور •1 أسئلة وتم اختبارها إحصائيا من خلال: اختبار ألفا كرونباخ Cronbach's alpha لقياس ثنات وصدق محتوى استيان الدراسة. حيث يوضح مدى أمكانية الاعتمادية على نتائج قائمة الاستقصاء، ومدى إمكانية 
تعميم نتائجها على مجتمع الدراسة، حيث إن معامل ألفا يقع بين (•، ( ) فإذا كانت قيمة ألفا

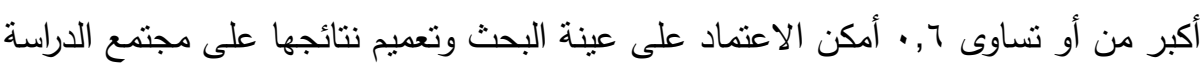

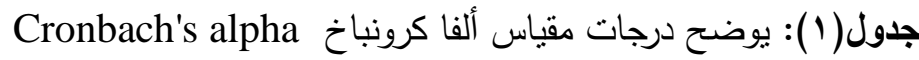

\begin{tabular}{|c|c|}
\hline درجة القبول & كرونباخ \\
\hline ممتاز & $\cdot, .9 \alpha \geq$ \\
\hline جيد & $\cdot, q>\alpha \geq \cdot, v$ \\
\hline مقبول & $\cdot, v>\alpha \geq \cdot, 7$ \\
\hline فقير & $\cdot, 7>\alpha \geq \cdot, 0$ \\
\hline غير مقبول & $\cdot, 0>\alpha$ \\
\hline
\end{tabular}

صدق وثبات درجات الأداة: تعتبر الأداة صادقة إن كانت تقبس ما وضعت لقياسه، أو الصفة

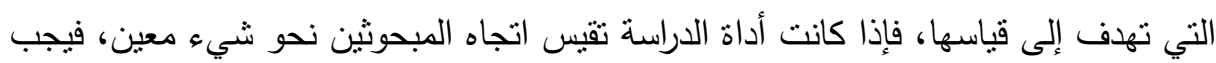

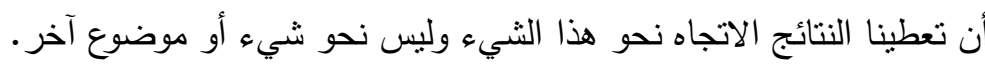

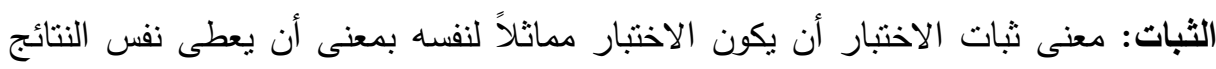

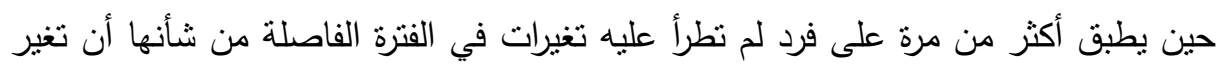
من الظاهرة التي يقيسها الاختبار • ويوضح ذلك مدى مدى إمكانية الاعتمادية على نتائج قائمة الاستبيان ، ومدى إمكانية تعميم نتائجها على مجتمع الدراسة.

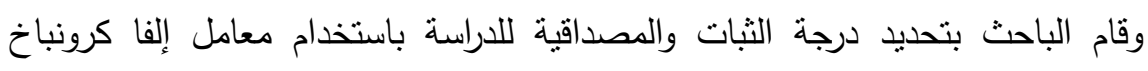
للتحقق من ثبات الأداة والاتساق الداخلي للتحقق من الصدق ويتضح ذلاتك من الجدول رقم

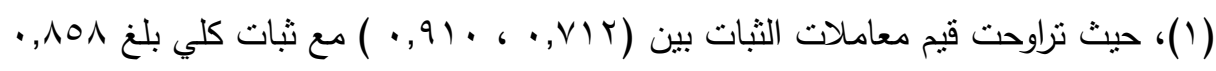

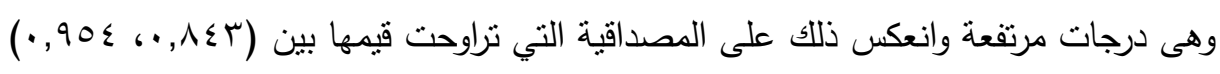

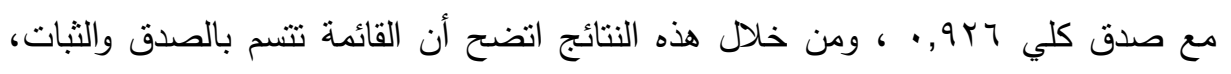

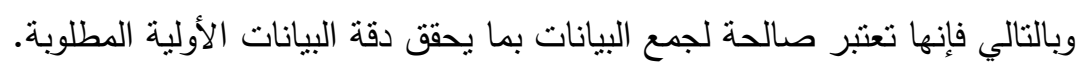

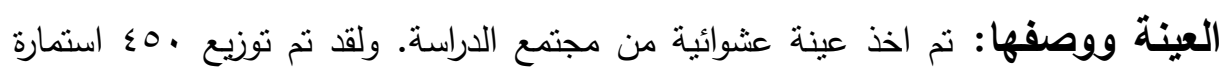
واستبعد بT استمارة من الاستمارات، ذلك بسبب عدم إجابة بعض المستقصي منهم على

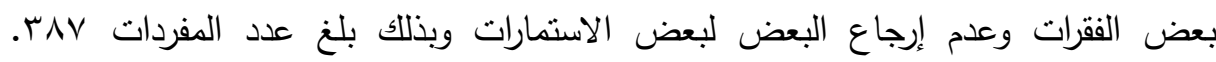


والجدول التالي يوضح توزيع عينة الدراسة ووصفها حيث تم وصف عينة الدراسة بناءً على عدد من الخصائص الديمجرافية التالية (الوظيفة- المؤهل - مدة الخبرة الوظيفية ). وفيما يلي عرض تقصيلي لها: جدول(ץ): يوضح توزيع عينة الدراسة طبقاً للمؤهل

\begin{tabular}{|c|c|c|}
\hline$\%$ & S & المؤهل \\
\hline$\Lambda, \Gamma$ & rT & متوسط \\
\hline$V, Y$ & rA & فوق متوسط \\
\hline$V 0,0$ & YqY & جامعى \\
\hline 9, & ro & دراسات عليا \\
\hline $1 \ldots$ & rAV & الاجمالي \\
\hline
\end{tabular}

يوضح الجدول السابق توزيع عينة الدراسة البالغ عددها (rNv) مفردة نم توزيعها بنسبة

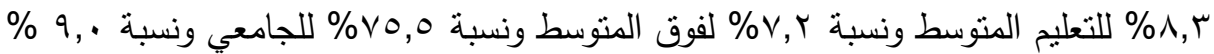

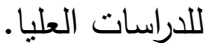

جدول(r): يوضح نوزيع عينة الدراسة طبقاً للوظيفة

\begin{tabular}{|c|c|c|}
\hline$\%$ & 5 & الوظيفة \\
\hline $0 \leqslant, \Gamma$ & YI. & مراجع داخلى \\
\hline$\varepsilon 0, V$ & $11 \mathrm{~V}$ & مراجع خارجى \\
\hline $1 \ldots$ & TAV & الإجمالي \\
\hline
\end{tabular}

يوضح الجدول السابق توزيع عينة الدراسة البالغ عددها (rNv) مفردة تم توزيعها بنسبة

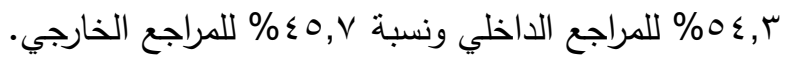
جدول(؛ ) : يوضح نوزيع عينة الدراسة طبقاً لسنوات الخبرة الوظيفي

\begin{tabular}{|c|c|c|}
\hline$\%$ & ك & الفئةًة \\
\hline$\varepsilon, \mathrm{V}$ & 11 & آقل من 0 سنوات \\
\hline$\varepsilon \vee, \cdot$ & IAY & من 0-. سنوات \\
\hline$r 0,1$ & $1 \% 4$ & 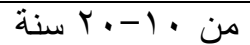 \\
\hline $1 T, Y$ & 01 & من · rاسنة فأكثز \\
\hline $1 \ldots$ & rAV & الإجمالي \\
\hline
\end{tabular}

يوضح الجدول السابق توزيع عينة الدراسة البالغ عددها (rNv) مفردة تم توزيعها بنسبة

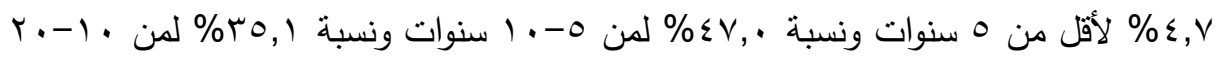

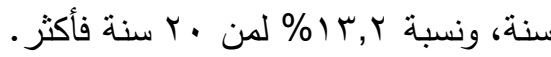




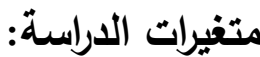

تتمنل متغيرات الدراسة فى متغيرين هما: المتغير المستقل: ويتكون من متغير واحد هو المراجعة الخارجية. المتغير التابع: ويتكون من متغير واحد هو هيكل الرقابة الداخلية.

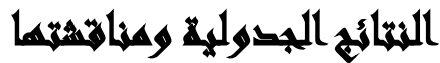

الفرض الأول: لا توجد فروق ذات دلالة معنوية بين إدراك فئات الدراسة لأهمية الإسناد الخارجي في الثكل الحالي لتقرير مراقب الحسابات الخارجي لتلبية احتياجات مستخدمي - مالتقارير جدول(ه): يوضح الاختلاف بين إدراك فئات الدراسة لأهمية الإسناد الخارجي في الثنكل

\begin{tabular}{|c|c|c|c|c|c|}
\hline \multirow{2}{*}{ الدالئي } & \multirow{2}{*}{ قيمة ت } & \multicolumn{2}{|c|}{ مراقب الحسابات الخارجى } & \multicolumn{2}{|c|}{$\begin{array}{c}\text { المراجع الإخلى } \\
\text { r. } \\
\end{array}$} \\
\hline & & الالمعراف & المتوسط & الالمعراف & المتوسط \\
\hline$\cdot, \cdot \leq 7$ & $r, \ldots l$ & $\cdot, \sum V$ & $r, v \cdot$ &., 49 & $r, 7)$ \\
\hline
\end{tabular}

تتثير بيانات الجدول السابق إلى وجود فرق دال إحصائيا بين إدرالك فئات الدراسة لأهمية الإسناد الخارجي في الثكل الحالي لتقرير مراقب الحسابات الخارجي لتلبية احتياجات مستخدمي الثقارير عند مستوى دلالة اقل من ه ..,.. حيث المراجع لخارجي أكثر موافقة على فئ أهمية الإسناد الخارجي في الثكل الحالي لتقرير مراقب الحسابات الخارجي لتلبية احتباجات

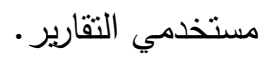

القرار هو عدم صحة فرض الدراسة القائل لا نوجد فروق ذات دلالة معنوية بين إدراك

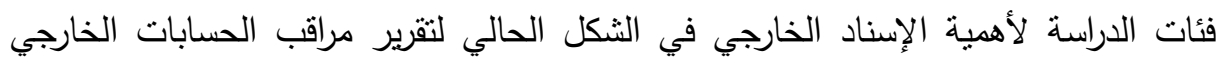
لتلبية احتباجات مستخدمي التقارير. 
الفرض الثاني: لا توجد فروق ذات دلالة معنوية بين آراء فئات الداسة حول تأثير تقرير مراقب الحسابات الخارجي عن الرقابة الداخلية البيئية على الثقة في تقريره التقليدي عن

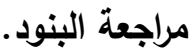
جدول(؟): يوضح الاختلاف بين آراء فئات الدراسة حول ثأثير تقرير مراقب الحسابات الخارجي عن الرقابة الداخلية البيئية على النقة في تقريره التقليدي عن مراجعة البنود التياه

\begin{tabular}{|c|c|c|c|c|c|}
\hline \multirow{2}{*}{ الدالئة } & \multirow{2}{*}{ قيمة ت } & \multicolumn{2}{|c|}{$\begin{array}{c}\text { مراقب الحسابات الخارجى } \\
\text { V v }\end{array}$} & \multicolumn{2}{|c|}{$\begin{array}{c}\text { المراجع الداخلى } \\
\text { r. }\end{array}$} \\
\hline & & الاتحراف & المتوسط & الانحراف & المتوسط \\
\hline . $\quad \leqslant V$ & 1,991 & 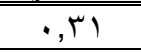 & $r, v 4$ & $\overline{e, T Y}$ & $\Gamma$, \\
\hline
\end{tabular}

تنثير بيانات الجدول السابق إلى وجود فرق دال إحصائيا حول تأثير تقرير مراقب الحسابات الخارجي عن الرقابة الداخلية البيئية على الثقة في تقريره التقليدي عن مراجعة البنود

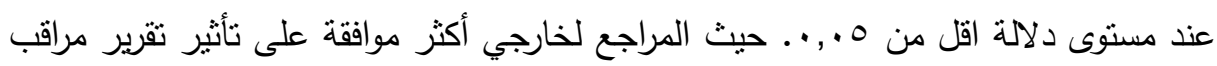

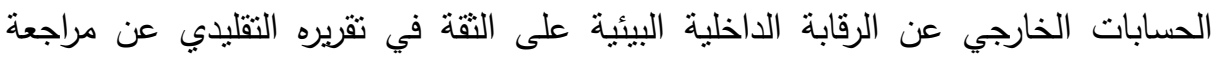

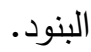

القرار هو عدم صحة فرض الدراسة القائل لا توجد فروق ذات دلالة معنوية بين آراء

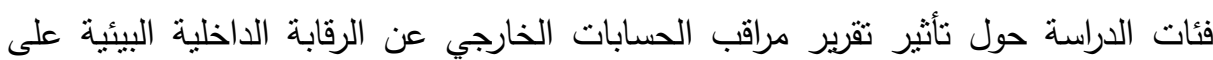
الثقة في تقريره التقليدي عن مراجعة البنود. الفرض الثالث: لا توجد فروق ذات دلالة معنوية بين آراء فئات الدراسة حول تأثير تقرير

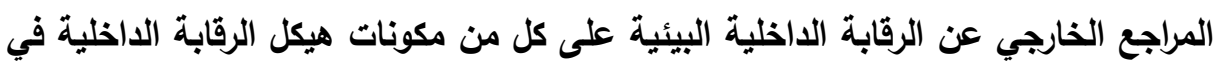
بيئة الأعمال المصرية. جدول (V): يوضح الاختلاف بين آراء فئات الدراسة حول ثأثثر تقرير مراقب الحسابات الخارجي عن الرقابة الداخلية البيئية على كل من مكونات هيكل الرقابة الداخلية

\begin{tabular}{|c|c|c|c|c|c|}
\hline \multirow{2}{*}{ مستوى الدلالة } & \multirow{2}{*}{ قيمة ت } & \multicolumn{2}{|c|}{ IVV= مراقب الحسابات الخارجين } & \multicolumn{2}{|c|}{ المراجع الداخلي م=. ا Y } \\
\hline & & الانحراف المعياري & المتوسط & الانحراف المعياري & المتوسط \\
\hline$\cdot, \cdot T \varepsilon$ & rצד & $\cdot, r q$ & r,or & $\cdot, r \varepsilon$ & $r, \Sigma \varepsilon$ \\
\hline
\end{tabular}

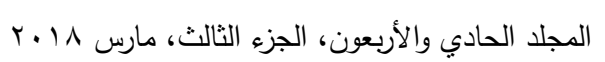


تشير بيانات الجدول السابق إلى وجود فرق دال إحصائيا حول تأثير تقرير مراقب الحسابات الخارجي عن الرقابة الداخلية البيئية على كل من مكونات هيكل الرقابة الداخلية في

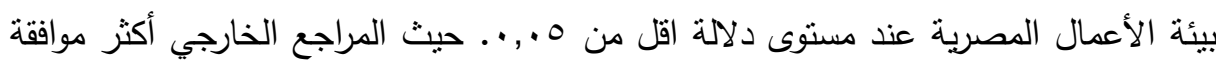
على تأثير تقرير مراقب الحسابات الخارجي عن الرقابة الداخلية البيئية على كل من مكونات هيكل الرقابة الداخلية في بيئة الأعمال المصرية.

القرار هو عدم صحة فرض الدراسة القائل لا توجد فروق ذات دلالة معنوية بين آراء

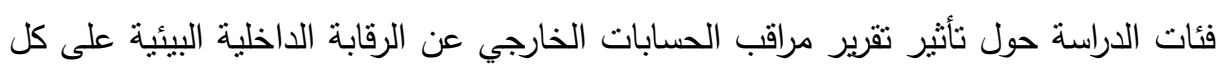
من مكونات هيكل الرقابة الداخلية في بيئة الأعمال المصرية.

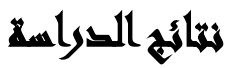

• لا تنتهي مراجعة الأداء البيئي بالثقرير عن النتائج انما منابعة نتائج المراجعة في أعداد

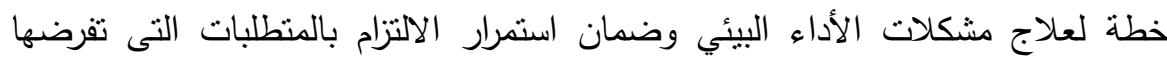
القوانين البيئية. • يجب أن يأخذ مراقب الحسابات الخارجي الآثار البيئية لعمليات التشغيل للمنثاة في الحسبان والتعويضات التي والغرامات المالية التي تتحملها المنشاة مقابل المخالفات البيئية. قام مراقب الحسابات الخارجي بتقديم التأكيد عن فعالية العمليات التي تقوم بها الإدارة لتحديد جميع المخاطر وتقييمها وترتيبها من شأنه أن يؤدى إلى تحسين فعالية إدارة - المخاطر ق قيام مراقب الحسابات الخارجي بمراجعة النظم الموضوعية للتأكد من الالتزام بالسياسات والخطط والإجراءات والقوانين البيئية لها تأثنر جوهري على كفاءة بيئة الرقابة.

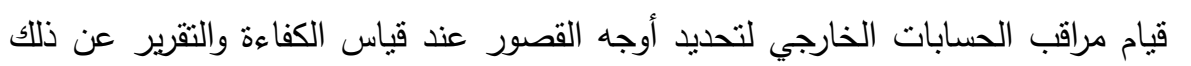
وتقديم التوصيات يؤدى الى تطوير كفاءة بيئة الرقابة.

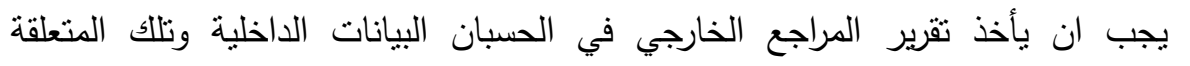
بالأحداث والأنشطة والظروف الخارجية والتشريعات البيئية التي تلتزم بها المنشاة في لئي الحسبان والتغيرات المستقبلية المحتملة في عمليات النتغيل. 


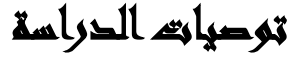

على ضوء الفرضيات والنتائج المتوصل إليها يمكننا اقتراح بعض التوصيات التي نساهم في تحسين آداء المراجعة:-

هجب نطوير تقرير المراجع الخارجي لتلبية احتياجات مستخدي التقرير •

لزوم التكامل الوظيفي بين المراجع الداخلي والخارجي لتحسين نظام الرقابة الداخلية. ضرورة إلزام المنشات بإعداد تقرير عن فعالية الرقابة الداخلية يشمل مكونات نظام الرقابة الداخلية التي نوجد فيها نقاط الضعف، ووصف نتلك النقاط اذا كانت هامة وما إذا كانت

$$
\text { تلقى الاهتمام المناسب من الإدارة. }
$$

• ضرورة الأخذ في الاعتبار تقرير مراقب الحسابات الخارجي والتتديد على المنشآت وخاصة الإدارة العليا ومراجعيها الداخليين تتفيذ تقرير مراقب الحسابات الخارجي وذلك للعمل على التطوير المستمر للنظام.

\section{zall}

محمد عبد الفتاح محمد: منهج مقترح للمراجعة البيئية بهدف زيادة فعالية تقرير مراقب

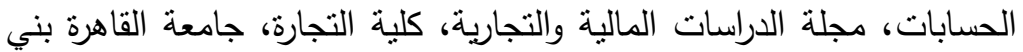

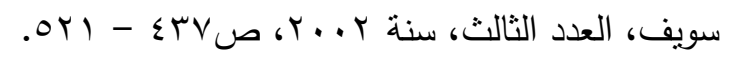

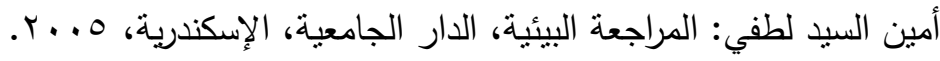

محمود يوسف الكاثف: اتجاهات تطوير القياس والإفصاح المحاسبي نحو نظام متكامل

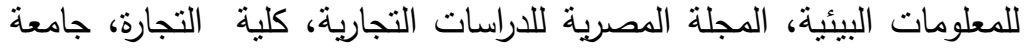

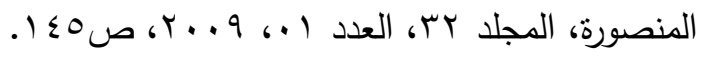

عبد السلام عبد اله ابو سرعة: التكامل بين المراجعة الداخلية والمراجعة الخارجية، رسالة

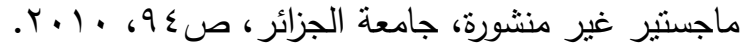

على حسين الدوغجى وأسامة عبد المنعم سيد: دور قانون (ساربنيز - اوكسيلى) فى رفع كفاءة

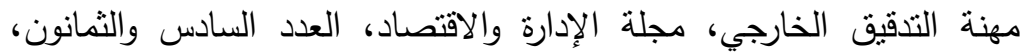

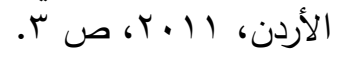


منال حامد فراج: أثر اختبار المصدر الخارجى لأداء وظائف المراجعة الداخلية على جودة

المراجعة وتخفيض تكاليف ادائها - دراسة ميدانية، المجلة العلمية التجارة

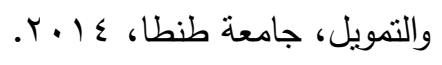

Beaumont, N. and Sohal (2004): Outsourcing in Australia, International Journal of Operations and Production management, PP.688700 .

Mehmet, Ozbirecikli (2007): A review on how CPAs (Certfied Public Accountants) Should Be Involved in Environmental Auditing and Reporting for the Core Aim of it "problems and perspectives in management", vol.5 , pp.113-126.

James, A. (2015): Objectivity and Independence: The Dual Roles of External Auditors and Forensic Accountants DiGabriele Montclair State University Marianne Ojo George Mason University; American Accounting Association.

\title{
THE IMPACT OF OUTSOURCING ON THE
}

\section{EFFECTIVENESS OF THE STRUCTURE OF THE}

ENVIRONMENTAL INTERNAL CONTROL

A FIELD STUDY

Mohamed, A. M. ${ }^{(1)}$; Adel, Dalia ${ }^{(1)}$ and Abdel Haleem, Safaa, M. 1) Faculty of Commerce, Ain Shams University

\begin{abstract}
This current study drives at exposing duties and responsibilities of accountants' controller in terms of common known auditing standards; exposing as well the significance of internal control system report and the difficulties the management may encounter to fulfill a report. It also explains the impact of internal auditor's report on efficacy of the

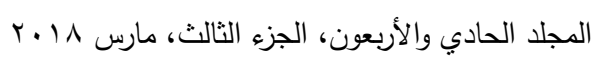


محمد عبد الفتاح وآخرون

environmental internal control structure. The researcher administers a field study using investigation of respondents' views of a sample of internal/external auditors to check out impact of audit external predication on effectiveness of the structure of environmental internal control.

Results indicate the necessity for functional integration between internal and external auditor to improve the internal control system and checking out efficancy of designing and operating the institution' control system due to standards and if it is appropriate for external auditor to fulfill his tasks. 